\title{
Enhancement of Segregation by Substrate Temperature Increase and Carbon Addition
}

\author{
H. Masuya and H. Awano (Sony Corp.)
}

(a)

(b)

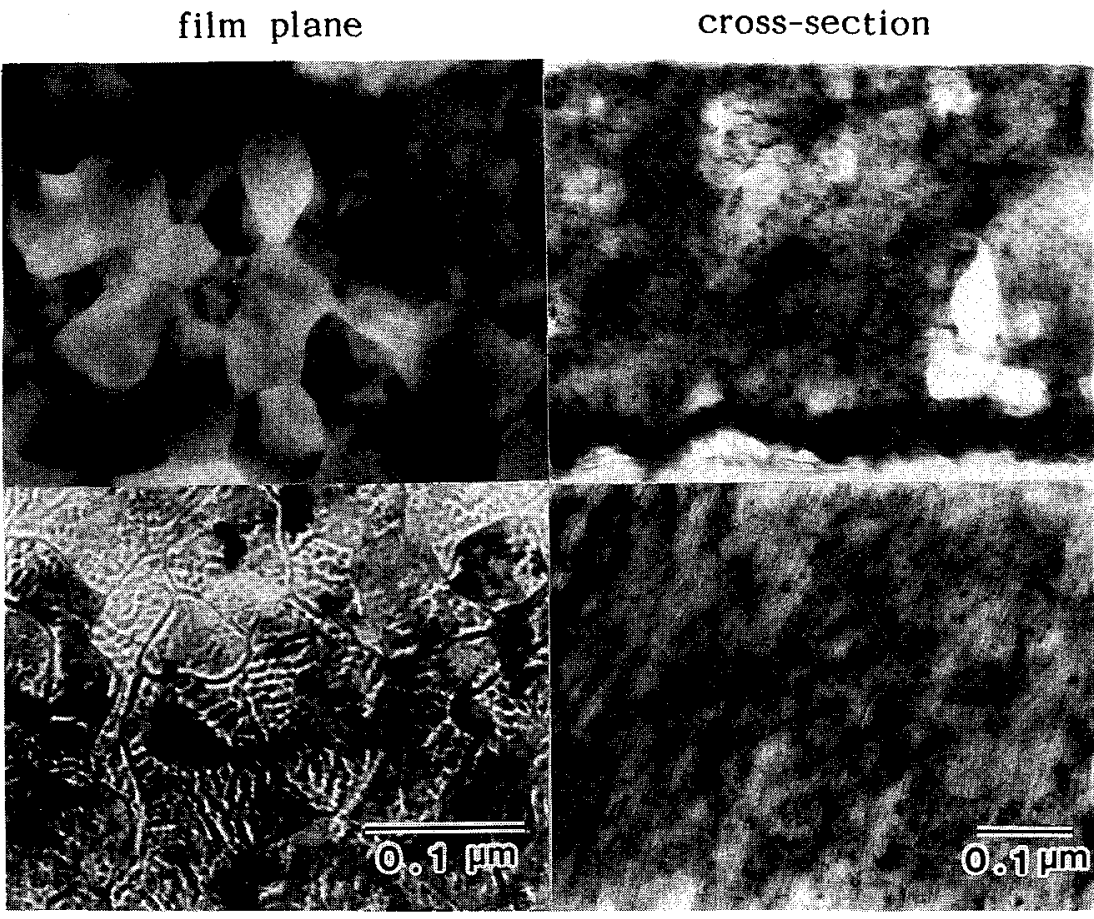

Compositionally segregated microstructures have a strong relationship to film magnetic properties. For sputter-deposited Co-23at\%Cr films, we find that both increasing the substrate temperature and adding carbon are very effective to enhance segregation, i.e. $\mathrm{Ms}, \mathrm{Hc}_{\perp}$ and perpendicular anisotropy. Changes in the segregated microstructures of the film plane and cross section are observed by TEM using the Maeda method. The conventional film in Photo (a) is deposited at a substrate temperature of $90^{\circ} \mathrm{C}$ without carbon addition, and the segregation-enhanced film in Photo (b) at $140^{\circ} \mathrm{C}$ with a carbon addition of $0.25 \mathrm{at} \%$. The bright and dark regions in the photos correspond to the Co-rich and $\mathrm{Cr}$-rich regions, respectively. Compared with the conventional film which has $\mathrm{Cr}-\mathrm{rich}$ regions only near grain boundaries, the segregation-enhanced film exhibits a network-like segregated microstructure which appears unrelated to the grain shape.

[Ref] H.Masuya and H.Awano, Jpn. J. Appl. Phys., 28 (1989) 372.

H.Masuya and H.Awano, IEEE Trans. Magn., MAG-23 (1987) 2064. 